\title{
Synthesis and Crystal Structure of a Sandwich-type Transition Metal Complex with Tungstobismutate and Triethanolamine
}

\author{
Xiuhong Xu, Ling Zhang, Yi Zhang, Bin Qi, and Fang Luo \\ Institute of Functional Polyoxometalate Chemistry, Faculty of Chemistry, \\ Northeast Normal University, Changchun, Jilin 130024, P. R. China \\ Reprint requests to Dr. Fang Luo. Tel: 86-431-85099667. E-mail: luof746@nenu.edu.cn
}

Z. Naturforsch. 2009, 64b, 821 - 825; received February 27, 2009

The sandwich-type complex $\mathrm{Na}\left(\mathrm{H}_{2} \mathrm{O}\right)_{6}\left(\mathrm{C}_{6} \mathrm{H}_{16} \mathrm{O}_{3} \mathrm{~N}\right)_{2}\left[\mathrm{Na}_{2}\left(\mathrm{H}_{2} \mathrm{O}\right)_{7}\left(\mathrm{C}_{6} \mathrm{H}_{16} \mathrm{O}_{3} \mathrm{~N}\right)\right]_{2}\left[\left(\mathrm{Na}\left(\mathrm{H}_{2} \mathrm{O}\right)_{2}\right)_{3}\right.$ $\left.\left(\mathrm{Cu}\left(\mathrm{H}_{2} \mathrm{O}\right)\right)_{3}\left(\mathrm{BiW}_{9} \mathrm{O}_{33}\right)_{2}\right] \cdot 14 \mathrm{H}_{2} \mathrm{O}(\mathbf{1})\left(\mathrm{C}_{6} \mathrm{H}_{16} \mathrm{O}_{3} \mathrm{~N}^{+}=\right.$protonated triethanolamine $)$has been synthesized and structurally characterized by single-crystal X-ray diffraction, IR spectroscopy and TG analysis. The basic framework of $\mathbf{1}$ is built from a sandwich-type $\left[\left(\mathrm{Na}\left(\mathrm{H}_{2} \mathrm{O}\right)_{2}\right)_{3}-\right.$ $\left.\left(\mathrm{Cu}\left(\mathrm{H}_{2} \mathrm{O}\right)\right)_{3}\left(\mathrm{BiW}_{9} \mathrm{O}_{33}\right)_{2}\right]^{9-}$ anion, two $\left[\mathrm{Na}_{2}\left(\mathrm{H}_{2} \mathrm{O}\right)_{7}\left(\mathrm{C}_{6} \mathrm{H}_{16} \mathrm{O}_{3} \mathrm{~N}\right)\right]^{3+}$ cations, a free $\mathrm{Na}\left(\mathrm{H}_{2} \mathrm{O}\right)_{6}{ }^{+}$ cation, and two free $\mathrm{C}_{6} \mathrm{H}_{16} \mathrm{O}_{3} \mathrm{~N}^{+}$units. In compound $\mathbf{1}$, the $\left[\left(\mathrm{Na}\left(\mathrm{H}_{2} \mathrm{O}\right)_{2}\right)_{3}\left(\mathrm{Cu}\left(\mathrm{H}_{2} \mathrm{O}\right)\right)_{3} 3^{-}\right.$ $\left.\left(\mathrm{BiW}_{9} \mathrm{O}_{33}\right)_{2}\right]^{9-}$ anion links two bi-nuclear units $\left[\mathrm{Na}_{2}\left(\mathrm{H}_{2} \mathrm{O}\right)_{7}\left(\mathrm{C}_{6} \mathrm{H}_{16} \mathrm{O}_{3} \mathrm{~N}\right)\right]^{3+}$ to form a $\left[\mathrm{Na}_{2}-\right.$ $\left.\left(\mathrm{H}_{2} \mathrm{O}\right)_{7}\left(\mathrm{C}_{6} \mathrm{H}_{16} \mathrm{O}_{3} \mathrm{~N}\right)\right]_{2}\left[\left(\mathrm{Na}\left(\mathrm{H}_{2} \mathrm{O}\right)_{2}\right)_{3}\left(\mathrm{Cu}\left(\mathrm{H}_{2} \mathrm{O}\right)\right)_{3}\left(\mathrm{BiW}_{9} \mathrm{O}_{33}\right)_{2}\right]^{3-}$ cluster, and a new 3D supramolecular structure is further constructed by hydrogen bonding interactions among this cluster, the free $\mathrm{Na}\left(\mathrm{H}_{2} \mathrm{O}\right)_{6}{ }^{+}$cation, and the free $\mathrm{C}_{6} \mathrm{H}_{16} \mathrm{O}_{3} \mathrm{~N}^{+}$cations. Crystal data: $\mathrm{Cu}_{3} \mathrm{Na}_{8} \mathrm{Bi}_{2} \mathrm{~W}_{18} \mathrm{C}_{24} \mathrm{H}_{150} \mathrm{O}_{121} \mathrm{~N}_{4}$, monoclinic, $C 2 / c, a=14.5668(13), b=23.0405(13), c=37.412(2) \AA, \beta=91.569(10)^{\circ}, Z=4$.

Key words: Sandwich, Tungstobismutate, Organic Ligand, Triethanolamine

\section{Introduction}

Polyoxometalates (POMs) [1-5], as one kind of significant metal oxide clusters with nanosizes and abundant topologies, have recently been employed as inorganic building blocks for 1D, 2D, or even 3D extended solid frameworks. Parallel to the rapid progress of POMs, particular attention has been devoted to families of transition metal-substituted POMs because of their highly tunable nature, coupled with their fascinating properties which lead to potential applications in catalysis [6,7], magnetism [8-11], and medicine [1214]. Among these, the sandwich-type species based on tri-vacant Keggin moieties probably represent the largest subunit owing to their defining of a planar minisurface.

Since the first sandwich-type polyanion $\left[\mathrm{Co}_{4}-\right.$ $\left.\left(\mathrm{H}_{2} \mathrm{O}\right)_{2}\left(\alpha-\mathrm{B}-\mathrm{PW}_{9} \mathrm{O}_{34}\right)_{2}\right]^{10-}$ was found by Weakley et al. in 1973 [15], many sandwich-type compounds have been synthesized. In addition, many investigations focused on tri-vacant tungstophosphate, -silicate, and -arsenate fragments. Up to now, the research for the analogs containing the subvalent main group atoms $\mathrm{Bi}^{\mathrm{III}}$ or $\mathrm{Sb}^{\mathrm{III}}$ as heteroatoms has been scarce. Known examples for such compounds with tungstobismutate include $\left[\left(\mathrm{CH}_{3}\right)_{4} \mathrm{~N}\right]_{6}\left[\mathrm{H}_{3} \mathrm{BiW}_{18} \mathrm{O}_{60}\right]$ [16],
$\left[\left(\mathrm{Mn}^{\mathrm{II}}\left(\mathrm{H}_{2} \mathrm{O}\right)_{3}\right)_{2}\left(\mathrm{WO}_{2}\right)_{2}\left(\mathrm{BiW}_{9} \mathrm{O}_{33}\right)_{2}\right]^{10-} \quad[17], \mathrm{Na}_{12}-$ $\left[\left(\mathrm{Cu}\left(\mathrm{H}_{2} \mathrm{O}\right)\right)_{3}\left(\mathrm{BiW}_{9} \mathrm{O}_{33}\right)_{2}\right] \cdot 29 \mathrm{H}_{2} \mathrm{O}$ [18], $\left[\mathrm{Bi}_{2} \mathrm{~W}_{20^{-}}\right.$ $\left.\mathrm{M}_{2} \mathrm{O}_{70}\left(\mathrm{H}_{2} \mathrm{O}\right)_{6}\right]^{(14-2 n)-}\left(\mathrm{M}=\mathrm{Co}^{\mathrm{II}}, \mathrm{Zn}^{\mathrm{II}}, \mathrm{Fe}^{\mathrm{III}}\right)$ [19], $\mathrm{K}_{11} \mathrm{H}\left[\left(\mathrm{BiW}_{9} \mathrm{O}_{33}\right)_{3} \mathrm{Bi}_{6}(\mathrm{OH})_{3}\left(\mathrm{H}_{2} \mathrm{O}\right)_{3} \mathrm{~V}_{4} \mathrm{O}_{10}\right] \cdot 25 \mathrm{H}_{2} \mathrm{O}$ [20], $\quad\left[\mathrm{M}^{n+}{ }_{3}\left(\mathrm{H}_{2} \mathrm{O}\right)_{x}\left(\mathrm{BiW}_{9} \mathrm{O}_{33}\right)_{2}\right]^{(18-3 n)-}\left(\mathrm{M}^{n+}=\right.$ $(\mathrm{VO})^{\mathrm{II}}, x=0$ and $\mathrm{M}^{n+}=\mathrm{Cr}^{\mathrm{III}}, \mathrm{Mn}^{\mathrm{II}}, \mathrm{Fe}^{\mathrm{III}}, \mathrm{Co}^{\mathrm{II}}, \mathrm{Ni}^{\mathrm{II}}$, $\left.\mathrm{Cu}^{\mathrm{II}}, x=3\right)$ [21], $\left(\mathrm{NH}_{4}\right)_{14} \mathrm{Na}_{4}\left[\left(\mathrm{~Np}_{3} \mathrm{~W}_{4} \mathrm{O}_{15}\right)\left(\mathrm{H}_{2} \mathrm{O}\right)_{3}{ }^{-}\right.$ $\left.\left(\mathrm{BiW}_{9} \mathrm{O}_{33}\right)_{3}\right] \cdot 62 \mathrm{H}_{2} \mathrm{O}[22], \mathrm{K}_{2} \mathrm{Na}_{6}\left(\mathrm{NH}_{4}\right)_{6}\left[(\mathrm{VO})_{2}-\right.$ $\left.\left(\mathrm{BiW}_{9} \mathrm{O}_{33}\right)_{2}\right] \cdot 41 \mathrm{H}_{2} \mathrm{O}[23], \mathrm{K}_{12}\left[(\mathrm{VO})_{3}\left(\mathrm{BiW}_{9} \mathrm{O}_{33}\right)_{2}\right]$ - $29 \mathrm{H}_{2} \mathrm{O}$ [24], etc. [25-29]. However, organic ligand-modified tungstobismutates are rare, and only imidazole- or imidazole derivative-based polyanions were reported to date [30-33].

In this paper, we chose triethanolamine as the organic ligand to modify the tungstobismutate component and obtained a novel sandwich-type compound $\mathrm{Na}\left(\mathrm{H}_{2} \mathrm{O}\right)_{6}\left(\mathrm{C}_{6} \mathrm{H}_{16} \mathrm{O}_{3} \mathrm{~N}\right)_{2}\left[\mathrm{Na}_{2}\left(\mathrm{H}_{2} \mathrm{O}\right)_{7}\left(\mathrm{C}_{6} \mathrm{H}_{16} \mathrm{O}_{3} \mathrm{~N}\right)\right]_{2^{-}}$ $\left[\left(\mathrm{Na}\left(\mathrm{H}_{2} \mathrm{O}\right)_{2}\right)_{3}\left(\mathrm{Cu}\left(\mathrm{H}_{2} \mathrm{O}\right)\right)_{3}\left(\mathrm{BiW}_{9} \mathrm{O}_{33}\right)_{2}\right] \cdot 14 \mathrm{H}_{2} \mathrm{O}(\mathbf{1})$.

\section{Results and Discussion}

\section{Structure description}

In compound $\mathbf{1}$, the sandwich-type polyoxoanion $\left[\left(\mathrm{Na}\left(\mathrm{H}_{2} \mathrm{O}\right)_{2}\right)_{3}\left(\mathrm{Cu}\left(\mathrm{H}_{2} \mathrm{O}\right)\right)_{3}\left(\mathrm{BiW}_{9} \mathrm{O}_{33}\right)_{2}\right]^{9-}$ consists of two tri-vacant $\left[\alpha-\mathrm{B}-\mathrm{BiW}_{9} \mathrm{O}_{33}\right]^{9-}$ moieties linked by 

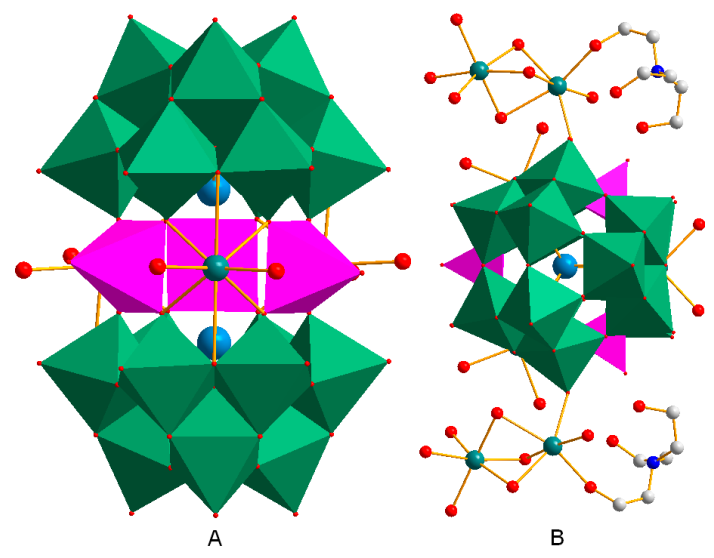

Fig. 1. (A) View of the $\left[\left(\mathrm{Na}\left(\mathrm{H}_{2} \mathrm{O}\right)_{2}\right)_{3}\left(\mathrm{Cu}\left(\mathrm{H}_{2} \mathrm{O}\right)\right)_{3}\right.$ $\left.\left(\mathrm{BiW}_{9} \mathrm{O}_{33}\right)_{2}\right]^{9-}$ polyanion, (B) view of the $\left[\mathrm{Na}_{2}\left(\mathrm{H}_{2} \mathrm{O}\right)_{7}\right.$ $\left.\left(\mathrm{C}_{6} \mathrm{H}_{16} \mathrm{O}_{3} \mathrm{~N}\right)\right]_{2}\left[\left(\mathrm{Na}\left(\mathrm{H}_{2} \mathrm{O}\right)_{2}\right)_{3}\left(\mathrm{Cu}\left(\mathrm{H}_{2} \mathrm{O}\right)\right)_{3}\left(\mathrm{BiW}_{9} \mathrm{O}_{33}\right)_{2}\right]^{3-}$ polyanion in the crystal structure of $\mathbf{1}$. Bi, sky blue; $\mathrm{Na}$, olive green; $\mathrm{O}$, red; $\mathrm{N}$, blue; $\mathrm{C}$, gray (color online).

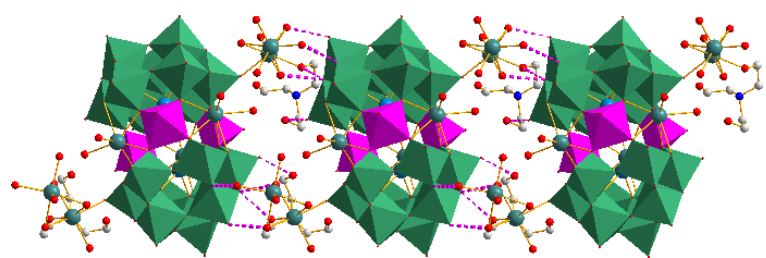

Fig. 2. A schematic illustration of the $1 \mathrm{D}$ chain of compound 1. The hydrogen bonds are labeled by light-purple dashed lines. $\mathrm{Bi}$, sky blue; $\mathrm{Na}$, olive green; $\mathrm{O}$, red; N, blue; $\mathrm{C}$, gray (color online).

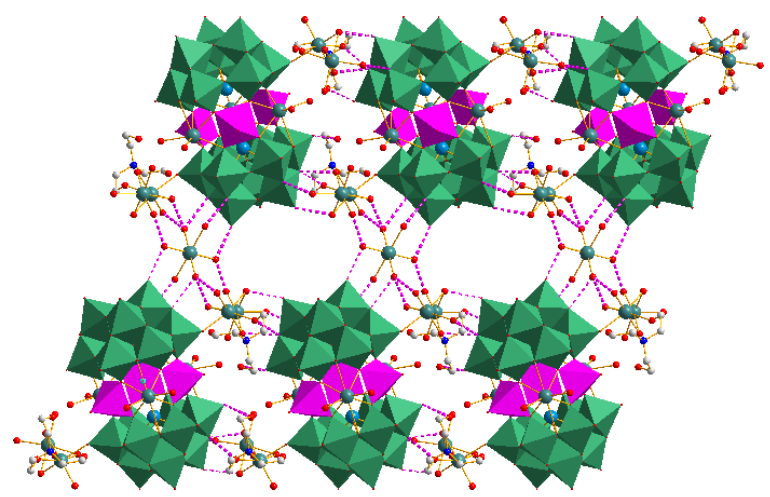

Fig. 3. View of the 2D layer of compound $\mathbf{1}$.

an alternating arrangement of three $\mathrm{Cu}^{2+}$ and three $\mathrm{Na}^{+}$ions (Fig. 1A). Three $\mathrm{Na}^{+}$ions and three $\mathrm{Cu}^{2+}$ ions are alternatingly linked to each other to form a six-membered ring, and these six atoms basically lie in the same plane. Each of the three equivalent $\mathrm{Cu}^{2+}$ ions exhibits a square-pyramidal coordination geometry defined by four $\mathrm{O}$ atoms
A

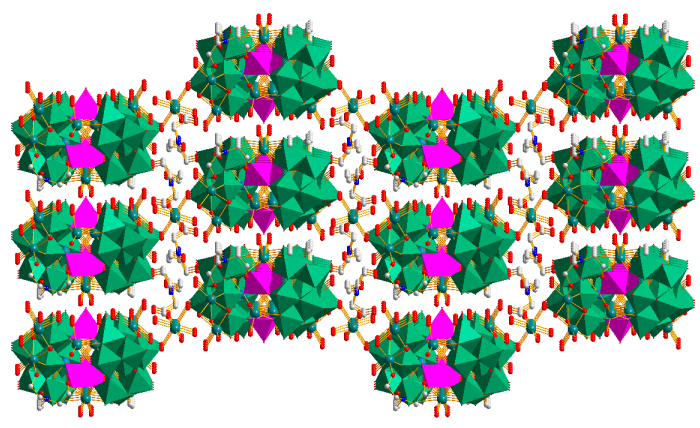

B

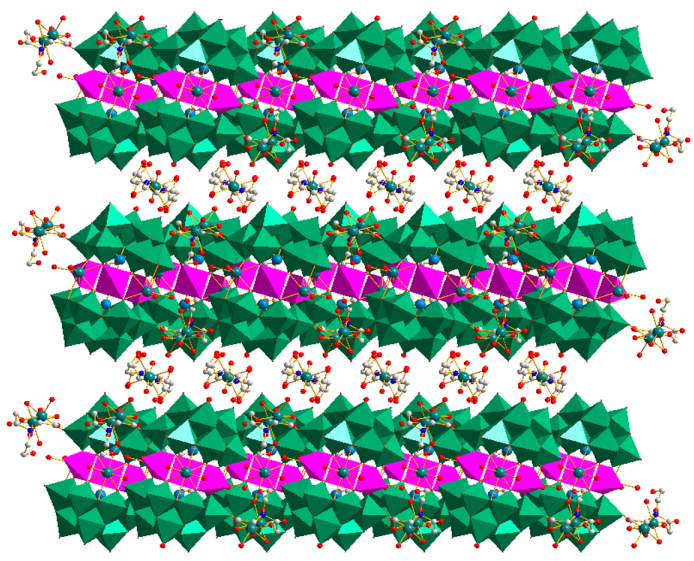

Fig. 4. Schematic illustration of the 3D framework of compound 1: (A) the extended structure viewed along the crystallographic $a$ axis, (B) the extended structure viewed along the $b$ axis.

from two tri-vacant $\left[\alpha-\mathrm{B}-\mathrm{BiW}_{9} \mathrm{O}_{33}\right]^{9-}$ subunits and one water molecule. The $\mathrm{Cu}-\mathrm{O}$ bond lengths are in the range of $1.926(7)-2.223(8) \AA$. A $\left[\mathrm{Na}_{2}-\right.$ $\left.\left(\mathrm{H}_{2} \mathrm{O}\right)_{7}\left(\mathrm{C}_{6} \mathrm{H}_{16} \mathrm{O}_{3} \mathrm{~N}\right)\right]_{2}\left[\left(\mathrm{Na}\left(\mathrm{H}_{2} \mathrm{O}\right)_{2}\right)_{3}\left(\mathrm{Cu}\left(\mathrm{H}_{2} \mathrm{O}\right)\right)_{3}(\mathrm{Bi}-\right.$ $\left.\left.\mathrm{W}_{9} \mathrm{O}_{33}\right)_{2}\right]^{3-}$ polyanion (Fig. 1B) is formed by a $\left[\left(\mathrm{Na}\left(\mathrm{H}_{2} \mathrm{O}\right)_{2}\right)_{3}\left(\mathrm{Cu}\left(\mathrm{H}_{2} \mathrm{O}\right)\right)_{3}\left(\mathrm{BiW}_{9} \mathrm{O}_{33}\right)_{2}\right]^{9-}$ anion connected with two $\left[\mathrm{Na}_{2}\left(\mathrm{H}_{2} \mathrm{O}\right)_{7}\left(\mathrm{C}_{6} \mathrm{H}_{16} \mathrm{O}_{3} \mathrm{~N}\right)\right]^{3+}$ units. In the $\left[\mathrm{Na}_{2}\left(\mathrm{H}_{2} \mathrm{O}\right)_{7}\left(\mathrm{C}_{6} \mathrm{H}_{16} \mathrm{O}_{3} \mathrm{~N}\right)\right]^{3+}$ unit, there are two crystallographically unique $\mathrm{Na}$ atoms. One $\mathrm{Na}$ atom adopts a distorted octahedral geometry, being coordinated by six water molecules, and the octahedron of the other $\mathrm{Na}$ atom is defined by one $\mathrm{O}$ atom of $\left[\alpha-\mathrm{B}-\mathrm{BiW}_{9} \mathrm{O}_{33}\right]^{9-}$, one $\mathrm{O}$ atom of a triethanolamine, and four of water molecules.

Fig. 2 shows that adjacent $\left[\mathrm{Na}_{2}\left(\mathrm{H}_{2} \mathrm{O}\right)_{7}\left(\mathrm{C}_{6} \mathrm{H}_{16}-\right.\right.$ $\left.\left.\mathrm{O}_{3} \mathrm{~N}\right)\right]_{2}\left[\left(\mathrm{Na}\left(\mathrm{H}_{2} \mathrm{O}\right)_{2}\right)_{3}\left(\mathrm{Cu}\left(\mathrm{H}_{2} \mathrm{O}\right)\right)_{3}\left(\mathrm{BiW}_{9} \mathrm{O}_{33}\right)_{2}\right]^{3-}$ anions are connected via hydrogen bonds formed by $\mathrm{O}$ atoms of coordinated water molecules and triethanolamine of $\left[\mathrm{Na}_{2}\left(\mathrm{H}_{2} \mathrm{O}\right)_{7}\left(\mathrm{C}_{6} \mathrm{H}_{16} \mathrm{O}_{3} \mathrm{~N}\right)\right]^{3+}$ units and of the terminal and bridging $\mathrm{O}$ atoms of the $[\alpha$ $\left.\mathrm{B}-\mathrm{BiW}_{9} \mathrm{O}_{33}\right]^{9-}$ anions. 1D chains are formed by these 
Table 1. Details of data collection and structure refinement for compound 1.

\begin{tabular}{ll}
\hline Formula & $\mathrm{Cu}_{3} \mathrm{Na}_{8} \mathrm{Bi}_{2} \mathrm{~W}_{18} \mathrm{C}_{24} \mathrm{H}_{150} \mathrm{O}_{121} \mathrm{~N}_{4}$ \\
$M_{\mathrm{r}}$ & 6533.28 \\
Crystal size, $\mathrm{mm}^{3}$ & $0.21 \times 0.16 \times 0.12$ \\
Crystal system & monoclinic \\
Space group & $C 2 / c$ \\
$a, \AA$ & $14.5668(13)$ \\
$b, \AA$ & $23.0405(13)$ \\
$c, \AA$ & $37.412(2)$ \\
$\beta$, deg & $91.569(1)$ \\
$V, \AA^{3}$ & $12551.70(15)$ \\
$Z$ & 4 \\
$D_{\text {calcd }}$, g cm & \\
$F(000), \mathrm{e}$ & 3.457 \\
Absorption coefficient, $\mathrm{mm}^{-1}$ & 11852 \\
$h k l$ range & 19.86 \\
& $-15 \leq h \leq 17,-26 \leq k \leq 27$, \\
$\theta$ range, deg & $-44 \leq l \leq 42$ \\
Reflections coll./unique $/ R_{\text {int }}$ & $1.65-25.00$ \\
Data/restraints/parameters & $31786 / 11056 / 0.042$ \\
$R 1 / w R 2[I \geq 2 \sigma(I)]$ & $11056 / 24 / 802$ \\
$R 1 / w R 2($ all data $)$ & $0.034 / 0.069$ \\
GoF $\left(F^{2}\right)$ & $0.047 / 0.072$ \\
Largest diff. peak/hole, e $\AA^{-3}$ & 1.06 \\
\hline & $1.25 /-2.57$ \\
\hline &
\end{tabular}

hydrogen bonds among different $\left[\mathrm{Na}_{2}\left(\mathrm{H}_{2} \mathrm{O}\right)_{7}\left(\mathrm{C}_{6}\right.\right.$ $\left.\left.\mathrm{H}_{16} \mathrm{O}_{3} \mathrm{~N}\right)\right]_{2}\left[\left(\mathrm{Na}\left(\mathrm{H}_{2} \mathrm{O}\right)_{2}\right)_{3}\left(\mathrm{Cu}\left(\mathrm{H}_{2} \mathrm{O}\right)\right)_{3}\left(\mathrm{BiW}_{9} \mathrm{O}_{33}\right)_{2}\right]^{3-}$ anions (Fig. 2).

In compound $\mathbf{1}$, there are also some $\mathrm{Na}$ atoms which are coordinated by six water molecules with octahedral coordination geometry. The $\mathrm{Na}\left(\mathrm{H}_{2} \mathrm{O}\right)_{6}{ }^{+}$ cations link the $1 \mathrm{D}$ chains by hydrogen bonds to form a 2D supramolecular structure (Fig. 3). Finally, a 3D extended framework is formed by hydrogen bonds among the different layers (Fig. 4). Acting as space-filling units, the free protonated triethanolamine molecules are intercalated in the interlayer voids via hydrogen bonds.

\section{IR spectrum of compound 1}

The IR spectrum of compound $\mathbf{1}$ has strong bands at 953,841 , and $758 \mathrm{~cm}^{-1}$ due to the characteristic absorption of $v\left(\mathrm{~W}-\mathrm{O}_{\mathrm{d}}\right), v\left(\mathrm{~W}-\mathrm{O}_{\mathrm{b}}\right)$, and $v\left(\mathrm{~W}-\mathrm{O}_{\mathrm{c}}\right)$, respectively. Bands at 3430, 2920, 2860, 1620, and $1410 \mathrm{~cm}^{-1}$ are attributed to the triethanolamine molecules.

\section{Thermal analysis}

The TG curve of compound $\mathbf{1}$ can be divided into five stages. The first two steps in the range of $29-145{ }^{\circ} \mathrm{C}$ are ascribed to the loss of crystal water molecules, coordinated water molecules bound to
Table 2. Selected bond lengths ( $\mathrm{A})$ and angles (deg) for compound $\mathbf{1}^{\mathrm{a}}$.

\begin{tabular}{|c|c|c|c|}
\hline$\overline{\mathrm{Cu}}(1)-\mathrm{O}(1)$ & $1.926(7)$ & $\mathrm{Na}(2)-\mathrm{O}(10)$ & $2.880(7)$ \\
\hline $\mathrm{Cu}(1)-\mathrm{O}(19)$ & $1.977(6)$ & $\mathrm{Na}(2)-\mathrm{O}(3)$ & $2.848(7)$ \\
\hline$u(1)-O(45)$ & $2.187(10)$ & ) $\mathrm{Na}(3)-\mathrm{O}(21)$ & $2.391(8)$ \\
\hline $\mathrm{Cu}(2)-\mathrm{O}(18)$ & $1.931(6)$ & $\mathrm{Na}(3)-\mathrm{O}(46)$ & $2.407(10)$ \\
\hline $\mathrm{Cu}(2)-\mathrm{O}(33)$ & $1.944(6)$ & $\mathrm{Na}(3)-\mathrm{O}(44)$ & $2.430(9)$ \\
\hline $\mathrm{u}(2)-\mathrm{O}(25)$ & $1.953(7)$ & $\mathrm{Na}(3)-\mathrm{O}(43)$ & $2.467(9)$ \\
\hline$(2)-O(28)$ & $1.964(7)$ & $\mathrm{Na}(3)-\mathrm{O}(42)$ & $2.646(10)$ \\
\hline $\mathrm{u}(2)-\mathrm{O}(40)$ & $2.223(8)$ & $\mathrm{Na}(3)-\mathrm{O}(37)$ & $2.348(12)$ \\
\hline $\mathrm{a}(1)-\mathrm{O}(41)$ & $2.410(9)$ & $\mathrm{Na}(4)-\mathrm{O}(43)$ & $2.463(9)$ \\
\hline $\mathrm{a}(1)-\mathrm{O}(25)$ & $2.441(7)$ & $\mathrm{Na}(4)-\mathrm{O}(46)$ & $2.395(8)$ \\
\hline $\mathrm{Na}(1)-\mathrm{O}(18)$ & $2.473(7)$ & $\mathrm{Na}(4)-\mathrm{O}(47)$ & $2.403(9)$ \\
\hline $\mathrm{a}(1)-\mathrm{O}(24)$ & $2.868(7)$ & $\mathrm{Na}(4)-\mathrm{O}(48)$ & $2.408(9)$ \\
\hline $\mathrm{a}(2)-\mathrm{O}($ & $2.364(8)$ & $\mathrm{Na}(4)-\mathrm{C}$ & $2.389(8)$ \\
\hline $\mathrm{Na}(2)$ & $2.444(7)$ & $\mathrm{Na}($ & $2.385(9)$ \\
\hline $\mathrm{Na}(2)-\mathrm{O}(19)$ & $2.462(8)$ & $\mathrm{Na}($ & $2.395(11)$ \\
\hline $\mathrm{a}(2)-\mathrm{O}(1)$ & $2.481(7)$ & $\mathrm{Na}(5$ & $2.407(9)$ \\
\hline $\mathrm{a}(2)-\mathrm{O}(33)$ & $2.484(7)$ & $\mathrm{Na}(5)-\mathrm{O}(34)$ & $2.574(12)$ \\
\hline $\mathrm{Na}(2)-\mathrm{O}(38)$ & $2.510(9)$ & & \\
\hline (1)- -( & 87.4(3) & $\mathrm{O}(39)$ & $95.0(3)$ \\
\hline$(1)^{\# 1}-\mathrm{Cu}($ & $90.2(3)$ & $\mathrm{O}(21$ & $93.6(3)$ \\
\hline $\mathrm{O}(1)-\mathrm{Cu}(1)-\mathrm{O}$ & $97.5(2)$ & $\mathrm{O}(21)-\mathrm{Na}(3)$ & $78.8(3)$ \\
\hline $\mathrm{O}(45)-\mathrm{Cu}(1)-\mathrm{O}(19)$ & $99.4(2)$ & $\mathrm{O}(21)-\mathrm{Na}(3)$ & $86.3(4)$ \\
\hline $\mathrm{O}(18)-\mathrm{Cu}(2)-\mathrm{O}(28)$ & $88.3(3)$ & $\mathrm{O}(21)-\mathrm{Na}(3)-\mathrm{O}(44)$ & $110.8(3)$ \\
\hline $\mathrm{O}(28)-\mathrm{Cu}(2)-\mathrm{O}(33)^{\# 1}$ & 89.1(3) & $\mathrm{O}(46)-\mathrm{Na}(3)-\mathrm{O}(42)$ & 78.7(3) \\
\hline $\mathrm{O}(33)^{\# 1}-\mathrm{Cu}(2)-\mathrm{O}(25)^{\# 1}$ & $87.3(3)$ & $\mathrm{O}(42)-\mathrm{Na}(3)-\mathrm{O}(37)$ & $110.1(4)$ \\
\hline $\mathrm{O}(25)^{\# 1}-\mathrm{Cu}(2)-\mathrm{O}(18)$ & $90.2(3)$ & $\mathrm{O}(43)-\mathrm{Na}(3)-\mathrm{O}(37)$ & $105.2(4)$ \\
\hline $\mathrm{O}(33)^{\# 1}-\mathrm{Cu}(2)-\mathrm{O}(40)$ & $96.0(3)$ & $\mathrm{O}(43)$ & $73.9(3)$ \\
\hline $\mathrm{O}(25)^{\# 1}-\mathrm{Cu}(2)-\mathrm{O}(40)$ & $98.0(3)$ & $\mathrm{O}(49)-\mathrm{Na}(4)-\mathrm{C}$ & $89.8(3)$ \\
\hline $\mathrm{O}(28)-\mathrm{Cu}(2)-\mathrm{O}(40)$ & $101.5(3)$ & $\mathrm{O}(47)-\mathrm{Na}(4)-\mathrm{C}$ & $87.3(3)$ \\
\hline $\mathrm{O}(18)-\mathrm{Cu}(2)-\mathrm{O}(40)$ & $98.8(3)$ & $\mathrm{O}(46)-\mathrm{Na}(4)-\mathrm{O}$ & $100.5(3)$ \\
\hline $\mathrm{O}(25)-\mathrm{Na}(1)-\mathrm{O}(18)$ & $76.7(2)$ & $\mathrm{O}(42)-\mathrm{Na}(4)-\mathrm{O}(43)$ & 78.7(3) \\
\hline $\mathrm{O}(18)-\mathrm{Na}(1)-\mathrm{O}(24)$ & $59.8(2)$ & $\mathrm{O}(49)-\mathrm{Na}(4)-\mathrm{O}(43)$ & $164.8(3)$ \\
\hline $\mathrm{O}(24)-\mathrm{Na}(1)-\mathrm{O}(41)$ & $84.5(2)$ & $\mathrm{O}(47)-\mathrm{Na}(4)-\mathrm{O}(43)$ & 103.9(3) \\
\hline $\mathrm{O}(41)-\mathrm{Na}(1)-\mathrm{O}(41) \#$ & 94.1(4) & $\mathrm{O}(46)-\mathrm{Na}(4)-\mathrm{C}$ & 79.2(3) \\
\hline $\mathrm{O}(41)-\mathrm{Na}(1)-\mathrm{C}$ & $86.0(2)$ & $\mathrm{O}(48)-\mathrm{Na}(4)-\mathrm{C}$ & $94.5(3)$ \\
\hline $\mathrm{O}(41)^{\# 1}-\mathrm{Na}(1)-\mathrm{O}(18)$ & $84.9(3)$ & $\mathrm{O}(35)-\mathrm{Na}(5)-\mathrm{O}(36)$ & $87.8(4)$ \\
\hline $\mathrm{O}(41)^{\# 1}-\mathrm{Na}(1)-\mathrm{O}(24)$ & $86.9(2)$ & $\mathrm{O}(35)^{\# 2}-\mathrm{Na}(5)-\mathrm{O}(36)$ & $92.2(4)$ \\
\hline $\mathrm{O}(1)-\mathrm{Na}(2)-\mathrm{O}(28)$ & $76.5(2)$ & $\mathrm{O}(35)-\mathrm{Na}(5)-\mathrm{O}(34)^{\# 2}$ & $80.6(4)$ \\
\hline $\mathrm{O}(3)-\mathrm{Na}(2)-\mathrm{O}(39)$ & $90.4(3)$ & $\mathrm{O}(35)^{\# 2}-\mathrm{Na}(5)-\mathrm{O}(34)^{\# 2}$ & $99.4(4)$ \\
\hline $\mathrm{O}(33)^{\# 1}-\mathrm{Na}(2)-\mathrm{O}(19)^{\# 1}$ & $74.7(2)$ & $\mathrm{O}(36)-\mathrm{Na}(5)-\mathrm{O}(34)^{\# 2}$ & $100.8(3)$ \\
\hline $\mathrm{O}(38)-\mathrm{Na}(2)-\mathrm{O}(39)$ & $90.6(3)$ & $\mathrm{O}(36)-\mathrm{Na}(5)-\mathrm{O}(34)$ & $79.2(3$ \\
\hline $\mathrm{O}(39)-\mathrm{Na}(2)-\mathrm{O}(19)^{7}$ & $90.3(3)$ & & \\
\hline
\end{tabular}

a Symmetry transformations used to generate equivalent atoms: ${ }^{\# 1}-x, y,-z-1 / 2{ }^{\# 2}-x-3 / 2,-y+1 / 2,-z$.

$\mathrm{Na}$ atoms, and interstitial triethanolamine molecules. The observed weight loss $(10.67 \%)$ is in agreement with the calculated value $(10.08 \%)$. The final three continuous steps occurring in the range $170-584{ }^{\circ} \mathrm{C}$ are due to the loss of more strongly coordinated water molecules and the coordinated triethanolamine molecules of the anions $(20.70 \%$ observed, $21.01 \%$ calcd.).

In summary, a new 3D supramolecular structure $\mathbf{1}$ has been synthesized under moderate conditions. It is the first inorganic-organic hybrid material which was 
constructed by modifying tungstobismutate with the organic ligand triethanolamine.

\section{Experimental Section}

General methods and materials

All reagents were purchased commercially and used without further purification. Deionized water was used for the synthesis of the target compound. The infrared spectrum was recorded on a Nicolet 170SX FT-IR spectrophotometer with a $\mathrm{KBr}$ pellet in the $4000-400 \mathrm{~cm}^{-1}$ region. TG analysis was performed on a Netzsch STA 449C microanalyzer in an atmosphere of nitrogen with a heating rate of $10{ }^{\circ} \mathrm{C} \mathrm{min}-1$ from 25 to $800{ }^{\circ} \mathrm{C}$.

\section{Synthesis}

$\mathrm{Na}\left(\mathrm{H}_{2} \mathrm{O}\right)_{6}\left(\mathrm{C}_{6} \mathrm{H}_{16} \mathrm{O}_{3} \mathrm{~N}\right)_{2}\left[\mathrm{Na}_{2}\left(\mathrm{H}_{2} \mathrm{O}\right)_{7}\left(\mathrm{C}_{6} \mathrm{H}_{16} \mathrm{O} \mathrm{O}_{3} \mathrm{~N}\right)\right]_{2-}$ $\left[\left(\mathrm{Na}\left(\mathrm{H}_{2} \mathrm{O}\right)_{2}\right)_{3}\left(\mathrm{Cu}\left(\mathrm{H}_{2} \mathrm{O}\right)\right)_{3}\left(\mathrm{BiW}_{9} \mathrm{O}_{33}\right)_{2}\right] \cdot 14 \mathrm{H}_{2} \mathrm{O}(\mathrm{I})$

In a typical preparation, $1.65 \mathrm{~g}(5 \mathrm{mmol})$ of $\mathrm{Na}_{2} \mathrm{WO}_{4} \cdot 2 \mathrm{H}_{2} \mathrm{O}$ was dissolved in $50 \mathrm{~mL}$ of deionized water, and this solution was heated to $80{ }^{\circ} \mathrm{C}$ with stirring. A solution of $\mathrm{Bi}\left(\mathrm{NO}_{3}\right)_{3} \cdot 5 \mathrm{H}_{2} \mathrm{O}(0.243 \mathrm{~g}, 0.5 \mathrm{mmol})$ in $1 \mathrm{~mL}$ of $6 \mathrm{M} \mathrm{HCl}$ was added dropwise. If a light turbidity occurred, addition was discontinued until the solution was clear again before the next drop was added. Then $\mathrm{CuCl}_{2} \cdot 2 \mathrm{H}_{2} \mathrm{O}$ $(0.171 \mathrm{~g}, 1 \mathrm{mmol})$ and triethanolamine $(0.13 \mathrm{~mL}, 1 \mathrm{mmol})$ were added in succession. The final $\mathrm{pH}$ of the mixture was adjusted to 5.5, and the resulting solution was stirred and heated at $80{ }^{\circ} \mathrm{C}$ for half an hour. After it had cooled to r.t., the mixture was filtered to remove insoluble solids, and the filtrate was allowed to evaporate slowly. After a few days, grass-green columnar single crystals suitable for $\mathrm{X}$-ray diffraction were isolated. $\mathrm{Cu}_{3} \mathrm{Na}_{8} \mathrm{Bi}_{2} \mathrm{~W}_{18} \mathrm{C}_{24} \mathrm{H}_{150} \mathrm{O}_{121} \mathrm{~N}_{4}$ : calcd. $\mathrm{Cu} 2.92$, Na 2.82, Bi 6.40, W 50.65, C 4.41, H 2.31, $\mathrm{N}$ 0.86; found $\mathrm{Cu} 3.32$, Na 3.12, Bi 6.60, W 51.15, C 3.98, $\mathrm{H} 1.85, \mathrm{~N} 0.52$.

\section{Crystal structure determination}

Single crystal X-ray diffraction data for compound $\mathbf{1}$ were recorded on a Bruker Apex CCD diffractometer with graphite-monochromatized $\operatorname{Mo} K_{\alpha}$ radiation $(\lambda=0.71073 \AA)$ at $296 \mathrm{~K}$. Absorption correction was applied using a multiscan technique. The structure was solved by Direct Methods with SHELXS-97 [34] and refined by full-matrix leastsquares on $F^{2}$ (SHELXL-97 [35]). Some of the non-hydrogen atoms were refined isotropically to avoid non-positive definite displacement ellipsoids. Most of the hydrogen atoms attached to carbon atoms were fixed in ideal positions. Likewise, the hydrogen atoms attached to water molecules could not be located in difference Fourier maps. For that reason no information regarding hydrogen bonds involving the water molecules can be given. Additional details of data collection and structure refinement are listed in Table 1. Selected bond lengths and angles are listed in Table 2.

CCDC 691703 contains the supplementary crystallographic data for compound $\mathbf{1}$. They can be obtained free of charge from The Cambridge Crystallographic Data Centre via www.ccdc.cam.ac.uk/data_request/cif.

\section{Acknowledgement}

We thank the Training Fund of the NENU's Scientific Innovation Project (NENU-STC08008), the Analysis and Testing Foundation of Northeast Normal University, and the Science Foundation for Young Teachers of Northeast Normal University (No. 20080302).
[1] M. T. Pope, Heteropoly and Isopoly Oxometalates, Springer, Berlin, 1983.

[2] C. L. Hill, Chem. Rev. 1998, 98, 1-2.

[3] T. M. Anderson, W. A. Neiwert, K. I. Hardcastle, C. L. Hill, Inorg. Chem. 2004, 43, 7353-7358.

[4] V. Kortz, S. S. Hamzeh, N. A. Nasser, Chem. Eur. J. 2003, 9, 2945-2952.

[5] H. T. Evans, Jr., J. Am. Chem. Soc. 1948, 70, 1291 1292.

[6] D. Kumar, E. Derat, A. M. Khenkin, R. Neumann, S. Shaik, J. Am. Chem. Soc. 2005, 127, $17712-17718$.

[7] A. R. Howells, A. Sankarraj, C. Shannon, J. Am. Chem. Soc. 2004, 126, $12258-12259$.

[8] P. Mialane, C. Duboc, J. Marrot, E. Rivière, A. Dolbecq, F. Sécheresse, Chem. Eur. J. 2006, 12, 19501959.

[9] M. J. Manos, A. J. Tasiopoulos, E. J. Tolis, N. Lalioti, J. D. Woollins, A.Z. Slawin, M. P. Sigalas, T. A. Kabanos, Chem. Eur. J. 2003, 3, 695-703.
[10] H. Andres, J. M. Clemente-Juan, M. Aebersold, H. U. Gudel, E. Coronado, H. Buttner, G. Kearly, J. Melero, R. Burriel, J. Am. Chem. Soc. 1999, 121, $10028-$ 10034.

[11] J. M. Clemente-Juan, H. Andres, J. J. Borras-Almenar, E. Coronado, H. U. Gudel, M. Aebersold, G. Kearly, H. Buttner, M. Zolliker, J. Am. Chem. Soc. 1999, 121, $10021-10027$.

[12] X. H. Wang, J. F. Liu, M. T. Pope, Dalton Trans. 2003, $957-960$.

[13] D. A. Judd, J. H. Nettles, N. Nevins, J. P. Snyder, D. C. Liotta, J. Am. Chem. Soc. 2001, 123, 886-897.

[14] J. T. Rhule, C. L. Hill, D. A. Judd, R. F. Schinazi, Chem. Rev. 1998, 98, 327-358.

[15] T. J. R. Weakly, Dalton Trans. 1973, 341 - 346.

[16] Y. Ozawa, Y. Sasaki, Chem. Lett. 1987, 16, $923-$ 926.

[17] M. Bösing, A. Nöh, I. Loose, B. Krebs, J. Am. Chem. Soc. 1998, 120, $7252-7259$. 
[18] C. Rosu, D. Rasu, T. J. R. Weakly, J. Chem. Crystallogr. 2003, 33, $751-755$.

[19] I. Loose, E. Droste, M. Bösing, H. Pohlmann, M. H. Dickman, C. Rosu, M. T. Pope, B. Krebs, Inorg. Chem. 1999, 38, 2688 - 2694.

[20] B. Botar, T. Yamase, E. Ishikawa, Inorg. Chem. Commun. 2000, 3, 579-584.

[21] D. Rusu, C. Crăcium, A. L. Barra, L. David, M. Rusu, C. Rosu, O. Cozar, G. Marcu, Dalton Trans. 2001, $2879-2887$.

[22] R. Copping, A. J. Gaunt, I. May, C. A. Sharrad, D. Collison, M. Helliwell, O. D. Fox, C. J. Jones, Chem. Commun. 2006, $3788-3790$.

[23] Z. H. Xu, J. Liu, E. B. Wang, C. Qin, Q. Wu, Q. Shi, J. Mol. Struct. 2008, 873, $41-45$.

[24] T. Yamase, E. Ishikawa, K. Fukaya, H. Nojiri, T. Taniguchi, T. Atake, Inorg. Chem. 2004, 43, $8150-$ 8157.

[25] B. Botar, T. Yamase, E. Ishikawa, Inorg. Chem. Commun. 2001, 4, 551- 554 .

[26] M. H. Alizadeh, M. Mohadeszadeh, J. Clust. Sci. 2008 , $19,435-443$

[27] Y. J. Wang, H. H. Zhang, C. C. Huang, X. H. Yu, Q.Z.
Sun, R. Q. Sun, Chinese J. Struct. Chem. 2004, 23, 902-907.

[28] D. Drewes, E. M. Limanski, M. Piepenbrink, B. Krebs, Z. Anorg. Allg. Chem. 2004, 630, 58-62.

[29] C. Y. Sun, S.X. Liu, C. L. Wang, L.H. Xie, C. D. Zhang, B. Gao, E. B. Wang, J. Coord. Chem. 2007, 60, $567-579$.

[30] H. L. Wang, G. L. Xue, J. W. Wang, D. Q. Wang, J. Li, Q.Z. Shi, Acta. Chim. Sinica 2003, 61, 1839-1843.

[31] H. Liu, L. Xu, Y. F. Qiu, W. J. An, Y. N. Jin, B. B. Xu, Chem. J. Chinese U. 2006, 27, 1409-1412.

[32] H. Liu, L. Xu, G. G. Gao, Y.F. Li, N. Jiang, J. Mol. Struct. 2008, 878, $124-130$.

[33] H. Liu, C. Qin, Y.G. Wei, L. Xu, G. G. Gao, F. Y. Li, X.S. Qu, Inorg. Chem. 2008, 47, 41664172.

[34] G. M. Sheldrick, SHELXS-97, Program for the Solution of Crystal Structures, University of Göttingen, Göttingen (Germany) 1997.

[35] G. M. Sheldrick, SHELXL-97, Program for the Refinement of Crystal Structures, University of Göttingen, Göttingen (Germany) 1997; see also: G. M. Sheldrick, Acta Crystallogr. 2008, A64, 112-122. 